\title{
DE L'EXPLOITATION A LA COMMERCIALISATION DES MINERAIS DU CUIVRE : DEFICIT JURIDIQUE OU EXPLOITATION DE L'HOM ME PAR L'HOMME AU KATANGA
}

\author{
Par Musoya Mazuwa Alexis ${ }^{1}$
}

\section{PRESENTATION DU KATANGA, LE CHAMPS D'INVESTIGATION}

Le Katanga est l'une des provinces de la République Démocratique du Congo qui est ellemême cet Etat du Congo qui fut successivement l'Etat Indépendant du Congo (E.I.C.), Congo-belge, République Démocratique du Congo, République du Congo, Zaïre et République Démocratique du Congo(RDC) aujourd'hui.

Cet Etat a connu des transformations sous plusieurs formes allant de l'aliénation à la tentative de colonisation passant par des manœuvres politiques teintées d'une colonisation de fait sans réaliser une colonisation conventionnelle. ${ }^{2}$

Katanga alors, existe à l'époque près Léopoldienne ${ }^{3}$, Léopoldienne et post Léopold II avec un changement politique et juridique. De ce fait, il a existé comme Katanga indépendant, comme Shaba et Katanga en 1997.

Notre analyse structuro-fonctionnaliste et exégétique part d'un constat de l'exploitation minière dans les sites sous examen.

\section{L'INTERET DE LA REFLEXION}

La RDC a la plus importante main-d'œuvre d'exploitation minière artisanale au monde, estimée à deux millions de personnes, mais le manque de contrôle de ce secteur a contribué à la dégradation des terres et à la pollution. Les réserves minérales inexploitées de la RDC (estimées à 24'000 milliards dollars US) ont une importance stratégique pour l'économie mondiale. $^{4}$

Quelques 15 tonnes de mercure sont utilisées chaque année dans l'exploitation minière artisanale en RDC, faisant du pays la deuxième plus importante source d'émissions de mer-

1 Assistant à l'Université de Lubumbashi, Faculté de Droit, mail: alexismusoya@yahoo.fr

2 Nous sommes d'accord avec le Professeur BANZA MALALE MAKUTA lorsqu'il est démontrable que sur le plan de Droit, la colonisation de la RDC par la Belgique n'était qu'une colonisation de fait et non de droit car la Belgique étant neutre; cette nature juridique ne lui donnait pas la capacité d'avoir une colonie.

3 Le Katanga comme nom a existé avant Léopold II sous forme traditionnelle, c'est le village Katanga situé à plus de $83 \mathrm{Km}$ de Lubumbashi.

4 Programme des Nations-Unies pour l'environnement (PNUE),Etude sur la portée de la potentialité environnementale de la RDC, Kinshasa, le 10 Octobre 2011. P.2. 
cure en Afrique ${ }^{5}$. Besoin est aussi d'attirer l'attention des plusieurs sur les impacts environnementaux liés à l'exploitation artisanale.

\section{Impacts écologiques de l'exploitation artisanale}

En l'absence d'un encadrement, les exploitations artisanales conduisent à une destruction anarchique des sols et au déboisement des zones exploitées.

Dans ces gigantesques et parfois éphémères zones d'exploitation, le souci de l'environnement n'existe pas dans l'esprit des Creuseurs. Tout au plus, l'administration essaie de minimiser quelque peu les effets les plus graves sur la sécurité des exploitants. Mais ces mesures semblent dérisoires face aux conséquences de ces exploitations.

Certaines de ces conséquences dégradent certainement l'environnement depuis longtemps, mais ne sont découvertes aujourd'hui que grâce à la sensibilité 6 du milieu aux nuisances minières, ou n'apparaissent que maintenant en raison de la lenteur et des effets cumulatifs de certaines contaminations.

De par leur fréquence et en l'absence de tout effort pour une régénération et une revalorisation des sites exploités, l'exploitation minière artisanale peut avoir des conséquences environnementales majeures sur l'écosystème. Dans le contexte congolais et particulièrement dans les cites visités par nous des puits sont souvent abandonnés, et offrent ainsi le sol au ravinement et à des processus d'érosion intensive, aboutissant à une destruction totale du couvert végétal et diminuant ainsi étendues des terres à usage agricole.

Une question reste pertinente lorsque la loi portant principes fondamentaux relatifs à la protection de l'environnement insiste sur le fait que toute activité tendant à avoir des répercutions sur l'environnement doit être concernée.

Ce déséquilibre provoque, en plus, un sur-alluvionnement des vallées et leur asphyxie plus ou moins profonde. Ces processus sont quasiment irréversibles et peuvent devenir catastrophiques à l'échelle de quelques générations. Ces exploitations anarchiques peuvent provoquer des effets convergents et causer de graves perturbations dans le drainage naturel des cours d'eau.

\section{Impacts sociaux de l'artisanat minier}

L'afflux massif de populations diverses sur les sites, dû à l'appétit d'un enrichissement facile et rapide, entraîne en général, une dégradation rapide des mœurs sur la plupart des sites miniers.

Comment 1'Etat congolais ne peut arriver à se soucier d'encadrer sa population dans cette vie sans valeurs sociales acceptables?

5 Idem.

6 Cette sensibilité est entendue aussi en termes de niveau de savoir scientifique des personnes appelées à lutter contre les nuisances de toute formes. 
Dans des carrières visitées par nous ce sont des antivaleurs, la prostitution, l'usage de stupéfiants, l'escroquerie, le banditisme et même la criminalité, bref toute sorte de la délinquance, qui tendance à s'y développer. Ce danger est d'autant plus réel que, souvent, il y a une nette insuffisance d'infrastructures sociales élémentaires, notamment aux plans santé, éducation, sécurité et même l'administration publique. En outre, un autre type d'impacts négatifs concerne les fréquents accidents mortels dus, à la fois, à l'inexistence des équipements de protection et aux excavations désordonnées.

C'est vrai, pour ce qui concerne les risques d'accidents, ceux-ci sont d'autant plus élevés que, parfois, sur la base de certaines croyances erronées, ces accidents sont interprétés comme un tribut nécessaire à payer pour trouver le métal précieux.

\section{Lorsqu'il s'agit des finances publiques, l'Etat est toujours le plus perdant}

L'on observe actuellement un phénomène remarquable d'éclosions de petites entreprises sociales, qui dépendent pour la plupart de l'exploitation des ressources naturelles. ${ }^{7}$

Cependant, du fait de la fragilité du système bancaire et du manque d'incitations à formaliser les transactions, ce secteur informel, échappant aux normes du travail et environnementales, s'est fortement développé et représente désormais un problème structurel majeur.

Même les entreprises minières ne veulent pas contribuer à cet aspect des choses qui inquiète le monde entier.

Derrière un visage angélique, celles-ci révèlent des agissements peu dignes du développement durable qu'elles disent vouloir promouvoir et se retrouvent souvent à la base de conflits sociaux récurrents.

Véritable «otage de la pauvreté», la population est en effet, contrainte de brader ses forêts pour quelques miettes, jusqu'au jour où elle se révolte et revendique ses droits, non sans répression.

\section{La gestion durable des forêts congolaises dans l'artisanat minier}

Nous n'allons pas citer les entreprises mais dans nos visites les cités de Fungurume, Kakanda, Kambove, Kipushi, Sakania, Luisha et les Villes de Likasi, kolwezi et Lubumbashi témoignent sur la question.

Si toutes les entreprises ne sont pas à mettre dans le même sac, une gestion viable économiquement, mais surtout respectueuse de l'environnement et des populations locales et autochtones, doit être mise en place le plus rapidement possible dans le plein respect des cadres légal et institutionnel sans complaisance.

7 Plusieurs quincailleries, selon le témoignage, ont vu le jour avec l'avènement de l'exploitation minière artisanale encouragée après la deuxième République. 


\section{LE DROIT POSITIF CONGOLAIS FACE A LA QUESTION}

Les engagements de la RDC en matière d'environnement sont clairement établis dans les textes constitutionnels.

Dans la Constitution de la transition du 04 avril 2003 et dans celle de 2006 soumise au référendum les articles suivants précisent :

- Art. 54 de la Constitution de la transition «Tous les Congolais ont droit à un environnement sain et propice à leur épanouissement.»

- «Les pouvoirs publics et les citoyens ont le devoir d'assurer la protection de l'environnement dans les conditions définies par la loi».

- Art. 53 du projet de Constitution «Toute personne a droit à un environnement sain et propice à son développement intégral» - «Elle a le devoir de la défendre» - «L'État veille à la protection de l'environnement et à la santé des populations» - «Les conditions de construction d'usines, de stockages, de manipulation, d'incinération des déchets toxiques, polluants ou radioactifs provenant des unités industrielles ou artisanales installées sur le territoire national sont fixées par la loi.» - «Toute pollution ou destruction résultant d'une activité économique donne lieu à compensation et/ou à réparation.»

- L'article 54 de la Constitution du 18 Fév. 2006, «la loi détermine la nature des mesures compensatoires, « réparatoires », ainsi que les modalités de leur exécution »

- Pour l'article 55 de la Constitution actuelle « Le transit, l'importation, le stockage, l'enfouissement, le déversement dans les eaux continentales et les espaces maritimes sous juridiction nationale, l'épandage dans l'espace aérien des déchets toxiques, polluants radioactifs ou de tout autre produit dangereux en provenance ou non de l'étranger, constitue un crime puni par la loi ».

- A propos de la loi n¹1/009 du 09 Juillet 2011 portant principes fondamentaux relatifs à la protection de l'environnement, elle est destinée à définir les grandes orientations en matière de protection de l'environnement;

- Orienter la gestion de l'immense potentiel dont dispose la RDC en ressources naturelles, dans la perspective d'un développement durable au profit de sa population;

- Prévenir les risques et lutter contre toutes les formes de pollutions et nuisances;

- Servir de socle aux législations spécifiques régissant la conduite des secteurs certes distincts de l'environnement mais dont les incidences directes sont indéniables.

Cette loi s'inspire essentiellement des principes fondamentaux et universels notamment :

- Le principe de développement durable;

- Le principe de la prévention et de correction;

- Le principe d'information et de participation du public au processus de prise des décisions en matière d'environnement;

- Le principe de précaution;

- Le principe d'intégration;

- Le principe de pollueur payeur;

- Le principe de coopération entre Etats en matière d'environnement. 
Elle s'articule autour de 9 chapitres que voici :

1. Des dispositions générales qui sont constituées des articles $1^{\text {er }}$ à 14 ;

2. Du cadre institutionnel qui comprend les articles 15à18;

3. Des mécanismes procéduraux, de l'article 19à24;

4. Des mécanismes de financement, 25à26;

5. De la gestion et de la conservation des ressources naturelles, de l'article 27à36;

6. De la prévention des risques et de la lutte contre les pollutions et nuisances, les articles 37 à 67 ;

7. De la responsabilité civile, les articles 68 à70;

8. Des infractions et des peines, les articles 71à 84;

9. Des dispositions transitoires, abrogatoires et finales, les articles 85 à89.

Toute pollution ou destruction résultant d'une activité économique donne lieu à compensation et/ou répartition ${ }^{8}$. Ceux-ci est le résultat de la rencontre ayant produit le principe pollueur-payeur.

L'article 111 du code minier règlemente l'autorisation d'exploitation artisanale en ces termes : Les cartes d'exploitant artisanal sont délivrées par le Chef de Division Provinciale des Mines du ressort aux personnes éligibles qui les demandent et qui s'engagent à respecter la réglementation en matière de protection de l'environnement, [...] conformément aux modalités qui sont fixées par le Règlement Minier après en avoir pris connaissance.

L'article 112 prévoit les obligations du détenteur de la carte d'exploitant artisanal.

Le détenteur d'une carte d'exploitant artisanal doit respecter les normes en matière de sécurité, d'hygiène, d'utilisation de l'eau et de protection de l'environnement qui s'appliquent à son exploitation conformément à la réglementation en vigueur. Il doit indemniser les exploitants agricoles pour tout dommage engendré par son activité.

Le Règlement Minier fixe les modalités d'exécution des normes en matière de sécurité publique, de santé publique et d'environnement.

L'article 415 du règlement minier prévoit même un code de conduite du prospecteur en ce sens tout prospecteur s'engage à respecter le code de conduite du prospecteur dont le modèle est repris en Annexe III du présent Décret portant le dit règlement, comme partie de sa déclaration de prospection.

Le prospecteur minier ne peut réaliser ses opérations de prospection qu'en conformité avec le code de conduite du prospecteur.

Le prospecteur qui n'exécute pas les obligations du code de conduite du prospecteur s'expose au retrait éventuel de son attestation de prospection.

L'article 416 lui insiste sur le code de conduite de l'exploitant artisanal en ces mots :Conformément à l'article $112 \mathrm{du}$ Code Minier, tout exploitant artisanal est tenu de s'engager à respecter le code de conduite de l'exploitant artisanal dont le modèle est repris en Annexe V du Décret, comme partie de sa demande de carte d'exploitant artisanal.

8 Article 54 de la constitution du 18 Fév. 
Le détenteur de la carte d'exploitant artisanal ne peut réaliser les opérations d'exploitation que conformément au code de conduite de l'exploitant artisanal.

A défaut d'observer ce code de conduite, la carte d'exploitant artisanal lui est retirée.

Les Services Techniques Spécialisés du Ministère des Mines chargés de l'encadrement de l'artisanat minier assurent la formation des exploitants artisanaux en philosophie et techniques de protection de l'environnement dans le cadre des opérations d'exploitation artisanale des produits des mines et des carrières.

L'article 417 s'intéresse quant à lui à la contribution de l'exploitant artisanal aux coûts de réhabilitation de la zone d'exploitation artisanale. Le taux de cette contribution est fixé à $10 \%$ du montant fixé pour l'obtention de la carte d'exploitation artisanale.

En plus de ses obligations définies au code de conduite de l'exploitant artisanal, le détenteur de la carte d'exploitant artisanal est tenu de contribuer au fond de réhabilitation institué en vue de financer la réalisation des mesures d'atténuation et de réhabilitation des zones d'exploitation artisanale, le taux de cette contribution est fixé à 10\% du montant fixé pour l'obtention de la carte d'exploitant artisanal.

Le Code forestier contribue également à la valorisation de la biodiversité, à la protection de l'habitat naturel de la faune sauvage et au tourisme'.

Les règles régissant l'exploitation minière artisanale sont entre autre :

- La Recommandation n`82 de 1947 sur l'inspection du travail (mines et transports)

- La recommandation n82 de 1965 sur l'âge minimum (travaux souterrains). Artisanaux et de la petite mine.

- La recommandation n²125 de 1965 sur les conditions d'emplois des adolescents (travaux souterraine);

- Recueil de directives pratiques du BIT de 1991 sur la sécurité et la santé dans les mines à ciel ouvert;

- La convention $n^{\circ} 123$ de 1965 sur l'âge minimum (travaux souterraines)

- La convention n¹23 de 1965 sur l'examen médicale des adolescents (travaux souterraines);

- La convention n ${ }^{\circ} 148$ de 1977 sur le lieu du travail (pollution de l'air, bruit et vibrations)

- La convention n¹76 de 1995 sur la sécurité et l’hygiène dans les mines

- Le guide sur la sécurité et l'hygiène dans les petites mines à ciel ouvert (2001).

\section{LA JURISPRUDENCE EN LA MATIERE}

Dans nos investigations, nous avons constaté que le juge congolais n'est presque pas présent sur les questions épineuses d'environnement, de finances publiques, de santé, d'éducation, de mœurs, etc. Et ce, en rapport avec le territoire de Kambove, même les escroqueries sont traitées souvent sans trace par les OPJ. Il est probale qu'il n'est pas sollicité car il ne peut pas se saisir seul. Le problème se complique lorsque seul un OPJ compétent en matière

9 Loi n ${ }^{\circ} 011 / 2002$ du 29 aout 2002 portant code forestier, article 3. 
de l'environnement minier peut déclencher la procédure si pollution il y a selon le code minier.

\section{LES PROBLEMES A RESOUDRE}

L'exploitation minière artisanale présente des nombreux problèmes à savoir :

- L'expression exacte de l'absence de l'encadrement sanitaire par l'Etat aux groupes vulnérables (qui les sont notoirement), les démunis, les femmes et les enfants trouvés dans des concessions déjà attribuées aux tiers conformément à la loi.

- Dans la province, des conflits entre les exploitants miniers artisanaux et les exploitants légaux. ${ }^{10}$

- Les Creuseurs sont souvent exploités avec facilités et sous un regard passif de l'autorité.

- Les pollutions sont multiples dans les sites visités.

- Les rivières sont utilisées pour les lavages des minerais de cuivre ou les hétérogénites (cobalt, cuivre, zinc,...) alors que plus près ces mêmes rivières sont les principales sources d'eau de boisson des ménages.

- Les arbres sont abattus sans autres formes de mesure de réhabilitation de l'environnement;

- Il n'y a aucune étude d'impact environnemental même si la loi actuelle de 2011 sur la protection de l'environnement assujetti toutes les activités de l'homme tendant à avoir un impact sur l'environnement.

- Le plus grand défi est celui institutionnel.

L'Etat du Congo éprouve d'énormes difficultés pour vivre selon sa vocation. Les autres iraient dans le sens de la « bonne gouvernance » qui est à rechercher.

Par contre, pour cette réflexion, il s'agit de rechercher la mission sacrée de l'Etat du Congo à la lumière des éléments ayant existés dès sa conception dans le processus finalisé par l'acte général de Berlin, son organisation et son fonctionnement eu égard à l'exploitation minière artisanale à l'aune du champ sous examen.

Notre inquiétude en cherchant à se lancer dans le concept " bonne gouvernance » se justifie dans le fait que ce dernier entraine derrière lui toutes les idéologies, cultures et missions de la légalisation mondiale ou internationale des ordres des abominations par les ministres et représentants de la destruction du monde et des civilisations.

Le code minier ou la loi n ${ }^{\circ} \mathrm{OO} 7 / 2002$ du 11juillet 2002 portant code minier : Quant aux exigences sociales, le Code institue le principe de la responsabilité de plein droit du titulaire pour les dommages causés du fait de l'occupation du sol, et le principe d'indemnisation des occupants du sol par le titulaire du droit minier ou de carrières.

L'article 15 qui stipule ce qui suit, " le service chargé de la protection de l'environnement minier au sein du ministère des mines exerce, en coordination avec les autres organismes de l'Etat chargés de la protection de l'environnement, les prérogatives qui leurs sont

10 Les exploitants légaux sont ceux qui ont un titre d'exploitation minière conformément au code minier de 2002. 
dévolues par le présent code et par tout autre réglementation en matière de protection de l'environnement ${ }^{11}$.

Mais à l'article 16 le code minier insiste sur le fait que seuls les services de l'environnement minier ont qualité pour exercer les prérogatives y afférentes.

Tout en sachant que ces services de l'environnement minier agissent par des agents de l'administration publique qui touchent, lorsqu'ils sont inspecteurs provinciaux, cent dollars et un sac de farine, ce qui n'est intéressant d'en savoir plus sur les moins gradés.

Le code minier démontre que le législateur a imposé au requérant du Permis d'Exploitation de présenter, à l'appui de sa demande de Permis, une Etude d'Impact Environnemental (EIE), le Plan d'atténuation et de Réhabilitation (PAR) et un Plan de Gestion Environnementale de son Projet (PGEP).

Il est vrai que la structure de l'administration a été modifiée en conséquence, avec la création notamment d'une Direction de l'Environnement minier.

Analysant la question, le Code Minier ne fait aucune référence directe au Ministère de l'Environnement.

Notre inquiétude reste aigüe lorsque le Code Minier n'impose aucune contrainte efficace d'ordre environnemental. Il se limite au retrait de la carte ou du permis selon le cas. Ce qui ne comble pas les principes pollueur-payeur et de réparation.

De même, le Code créé un « Permis d'Exploitation de Petite Mine » différent de l'exploitation artisanale en ce sens que des procédés semi-industriels ou industriels sont utilisés, mais là encore, le Code n'impose aucune contrainte d'ordre environnemental. Alors que l'exploitation artisanale et industrielle dans les zones minières est à la base de la dégradation de l'environnement en RDC.

Le foncier congolais se trouve dans le cas de figure dans une « dualité des systèmes juridiques » les textes ayant été écrits dans « le respect des droits coutumiers mais sans définir clairement comme à l'époque léopoldienne sur la question ${ }^{12}$.

Le code forestier, le code agricole, le code minier et d'autres loi traitant sur la question sont restés anachroniques par conséquent inappropriées.

Selon l'analyse de l'exposé de motif de la Constitution il ressort clairement que la législation en la matière étant anachronique, il s'avère indispensable que, conformément à l'article 123 point 15 de la dite Constitution du 18 fév. 2006, la République Démocratique du Congo dispose d'une loi cadre. La loi n¹1/009 du 09 Juillet 2011 portant principes fondamentaux relatifs à la protection de l'environnement.

11 Les articles 15 et $16 \mathrm{~S}$ du code minier et 11 du règlement minier. Dans la pratique le service du ministère de l'environnement se prévaut déjà de la légalité de leur descente sur terrain sur base de la loi de 2011 alors que celui de l'environnement minier continue avec une possibilité de la double taxation ou la simple tracasserie administrative.

12 Dès l'entrée en vigueur de la loi de 1973 les droits des occupants des terres rurales (communauté locales, membres des communautés traditionnelles) sont des droits de jouissance régulièrement acquis sur ces terres et qui seront réglés par une ordonnance du Président de la République. 
Mais cela ne va pas sans poser problème lorsque nous sommes en présence du code minier qui continue à œuvrer avec toutes ces confusions.

Tout ce cafouillage de textes juridiques n'inquiète pas la réalité qui continue son bonhomme de chemin selon notre constat amer.

Analysant transversalement la loi portant protection de l'environnement face aux institutions compétentes nous nous rendons compte que cette loi que nous qualifions de béatitude juridique se révèle comme un lion édenté teinté d'un parfait mimétisme juridique (du moins justifié dans le système onusien).

Si les lois de deux générations des droits de l'homme continuent à vivre une hypocrisie sans pareil alors qu'elles sont approuvées par la population elle-même, que dire de ce qui n'est pas encore connu par elle? Ceci veut dire que le niveau d'instruction qui a une influence sur le niveau de l'application des textes de loi joue beaucoup dans l'exploitation minière artisanale.

Un autre obstacle institutionnel peut être entretenu pour longtemps dans l'interprétation des articles 5,17,21,22,24,38,42,45,47,48,50,54,60,61 et 66 de loi portant principes fondamentaux relatifs à la protection de l'environnement en RDC.

Dans cette mesure, chaque fois elle renvoie(in fine) tous les articles précités à un décret délibéré en conseil des ministres qui est appelé à en fixer la nature et les conditions d'application des dispositions concernées.

Cet aspect des choses neutralise la densité même de la loi du fait de la mauvaise connotation de la gouvernance des institutions du pays ${ }^{13}$.

Actuellement comme il y a longtemps lorsque nous visitons les sites d'exploitation minière artisanale officielle ${ }^{14}$ et officieuse ${ }^{15}$ notre constat reste amer en plein $21^{\mathrm{e}}$ siècle l'on se croirait à l'époque d'avant abolition et incrimination de l'esclavage. Ceci n'est pas à comprendre au sens classique de l'esclavage mais assimilé à une nouvelle forme d'esclavage qui ne veut pas dire ouvertement son nom.

En fait, il s'agit d'un travail d'esclave paradoxalement, nous pouvons qualifier ces jeunes et adultes hommes ou femmes de courageux ou courageuses selon le cas dans la mesure où ils (elles) n'ont pas accepté la réalité propre au tiers mondistes comme une fatalité.

Mais ils (elles) trouvent dans cette misère une opportunité de renier leur vie sans faire du mal ni à l'Etat ni immédiatement au prochain du moins avec des sérieux problèmes socio-environnementaux et sanitaires. Par ce qui précède, eu égard à la vocation naturelle d'un Etat il y a lieu de nous interroger si l'Etat congolais existe-t-il chez les artisanaux?

13 La santé, l'économique du citoyen ou de la population, ses aspirations pèsent moins pour un animateur administratif congolais qui n'est intéressé que par le poids politique en termes de possibilité de porter des armes.

14 Parmi les sites que nous avons visités, nous pouvons citer les carrières de Kasonga, Luisha (+ou-86 Km de Lubumbashi), Kipesé (+ou-50Km de Likasi), Ambakuku (+ou-55 Km de Likasi) Kifumpa (+ou-60 Km de Likasi) avec une affectation en zone d'exploitation minière artisanale.

15 Actuellement sous une autre forme d'exploitation minière sans être artisanale, sans une affectation en zone d'exploitation minière artisanale. 
Cette préoccupation nous pousse à analyser si un Etat normal pouvait accepter la situation d'une précarité inouïe que traversent les artisanaux congolais dans les carrières minières visitées à travers la démarche de notre investigation.

Notre questionnement est d'autant plus vrai. Cependant nous ne nions pas l'existence de l'Etat du Congo dans le sens strict du terme. C'est-à-dire un Etat pour qu'il existe il faut qu'il y soit l'existence de trois éléments constitutifs de celui-ci plus les deux éléments de forme notamment la population, le territoire, le gouvernement, la souveraineté et la reconnaissance.

Notre inquiétude doit se comprendre dans le fait de l'exigence d'un appareil gouvernement qui justifie qu'il n'y a Etat que lorsque le pouvoir est exercé sur une collectivité localisée au moyen de règles créées et appliquées selon des procédures régulières et constantes ${ }^{16}$.

Le souci est non seulement guidé dans une vision répressive en faveur de l'environnement, les finances publiques, des droits des générations futures ou le civisme en général, mais aussi dans le sens profond du mot Etat.

A notre approbation explicite, nous tenons pour évidentes par elles-mêmes les vérités suivantes : tous les hommes sont crées égaux; ils sont doués par le créateur de certains droits indéniables; parmi ces droits se trouve la vie, la liberté et la recherche du bonheur. Les gouvernements sont établis parmi les hommes pour garantir ces droits, et leur juste pouvoir émane du consentement des gouvernés.

Toutes les fois qu'une forme de gouvernement devient destructive de ce but, le peuple a le droit de la changer ou de l'abolir et d'établir un nouveau gouvernement en le fondant sur les principes et l'organisation en la forme qui lui paraîtront les plus propres à lui donner la sureté et le bonheur ${ }^{17}$.

De ce fait, la protection des artisanaux doit se présenter sous plusieurs forme allant de la prévention passant par l'établissement des conditions socio-économiques entrant dans la philosophie du bonheur tout en débouchant à une juste récompense sans oublier la justice distributive.

En leurs consciences, les artisanaux savent que le pays détient d'immenses potentialités très variées et présentes sur l'ensemble du territoire national ainsi que des capacités pour promouvoir une économie introvertie, intégrée et entièrement modernisée.

16 RIVIER, R., Droit international public, Ed.PUF, Paris,2012.P.230.

17 VINCENSINI, J.J. cité par MOLE GBAMANZANGO J.F, Réflexion sur quelques déclarations relatives aux droits de l'Homme dans l'histoire, in Revue Africaine de Droit canonique, vol. I, $\mathrm{n}^{\circ} 1$. Avril 2007, P.13. 
La grande contradiction qui place l'Etat du Congo (RDC) sur la liste en position très défavorable ${ }^{18}$ de pays selon sa gouvernance ${ }^{19}$ suscite un comportement réciproque dans le chef de la plupart des actuels artisanaux.

En d'autres termes, lorsque l'Etat ne remplit pas correctement sa mission selon les principes de la bonne gestion des biens publics et du respect des libertés et droits de l'homme y compris l'exploitation et la mise en valeur de toutes les ressources du pays au profit du peuple tout entier et dans l'intérêt général de la nation, une désobéissance peut être palpable dans la vie quotidienne de ses populations implicitement ou tacitement selon les convictions.

Le cas de la RDC est tel que les autorités politico-administratives peuvent se frotter les mains du fait non pas de la culture de la population au sens charnel du terme mais de la culture au sens spirituel du mot. Cette culture est aussi influencée par la maxime selon laquelle «... tout homme soit soumis aux autorités qui exercent le pouvoir, car il n'y a pas d'autorité que par le créateur et celles qui existent sont établies par lui. Ainsi, celui qui s'oppose à l'autorité se rebelle contre l'ordre voulu par Dieu, et les rebelles attireront la condamnation sur eux-mêmes ». ${ }^{20}$

L'autorité établie (agissant selon la volonté du créateur ou non) doit savoir qu'elle a un rôle très capital à jouer dans l'éducation ou la formation de la population concernée. De ce fait, tout ce qu'elle fait compte en commençant par ses gestes, son tempérament, ses paroles, ses jugements, ses décisions, savoir que son activité et son inactivité font parties des éléments d'interprétation sur lesquels doit se focaliser l'obéissance de ses gouvernés.

Tel est le cas pendant la II ${ }^{\text {ème }}$ République qui inspirait l'obéissance des Zaïrois sous le postulat de "Tobongisa". C'est-à-dire un citoyen moyen obéissant devait se voir, par le système de gestion de la chose publique autorisé implicitement à pouvoir survivre pas par le salaire mais en usant de sa position sociale sans tenir compte des principes du trésor public.

Les conséquences peuvent être résumées dans le fait que l'Etat et les citoyens perdent dans l'évolution des finances publiques.

A la même époque, la phrase célèbre inspirée dans les consciences de l'élite Zaïroise peut être condensée dans ceci : « le MPR n'a pas besoin d'intellectuels froids ».

C'est dire qu'intelligents, génies ou savants (professeur des universités) si vous ne dansez pas pour le MPR ou ne chantez, vous ne valez-rien pour le pays (Zaïre).

18 Plusieurs rapports de la Banque Mondiale soulignent l'inexistence de la RDC sur les listes favorables en termes de volonté de bonne gestion des affaires publiques.

19 La gouvernance selon nous doit se comprendre, pour la RDC, comme la mission sacrée de l'Etat du Congo. Il s'agit là d'une négation des idéologies de bonne gouvernance teintées d'une sorte de mission de légalisation des abominations dans le monde avec une justification aléatoire et précoce.

20 Romains 13,1-7. Cette conception peut constituer une victoire puissante sur toute sorte de mal. Cependant, la moindre distraction volontairement acceptée continuellement entraine à la ruine pour des générations. 
Par ce fait, lorsqu'on est partisan absolu de l'obéissance sacrée cela pousse facilement et d'une façon latente à la perte totale ou partielle du goût de l'excellence ou de la méritocratie.

Par conséquent, la société est vouée à une dégradation fatale et un renversement des antivaleurs à la place des vertus et valeurs sociales.

Telle est la justification de la corruption, des détournements impunis, des promotions des courageux (c'est-à-dire selon le système, ceux qui sont en mesure de rompre avec le vrai, le bien, le juste, l'agréable, l'honnêteté, l'impartialité...).

Une autre approche, celle des Elites résistant ${ }^{21}$ au piège de la destruction, avait eu gain de cause en organisant la conférence nationale souveraine.

Bien sûr, jouer ce rôle n'est pas donné à tout le monde, même pendant la période d'avant Jésus-Christ les hommes qui avaient la vocation de Jérémie n'apparaissaient pas au nombre de mille au même moment. Mais tout le monde a l'obligation de résister selon son statut social et selon l'opportunité.

Dans cette optique, une nouvelle vision d'Elite a réussi à démontrer qu'il ne s'agissait pas des gouvernants efficaces mais de plafonnés malveillants.

Malheureusement, entre la dégradation totale du système de gouvernement et la désapprobation officielle de celui-ci par l'Elite de vocation, il s'était déjà installé la « mauvaise gouvernance » avant la $3^{\mathrm{e}}$ République, c'est presque une génération.

Une gangrène qu'il faut démanteler lorsque la construction est difficile par rapport à la destruction.

Comment y parvenir? Est-ce les gouvernants actuels sont dans la rupture ou dans le retour ou le recours au mobutisme pour espérer l'installation des vertus et de la bonne gestion ou la mission sacrée de l'Etat du Congo?

C'est vrai « là où l'homme est nié, le diable lui- même ne trouve pas son compte $»^{22}$. C'est de cette façon que nous pouvons analyser comment l'Etat ne gagne rien lorsqu'il s'agit de renforcer le trésor public.

En somme, souligne Monseigneur RUVEZI, évêque de Sakania-Kipushi, la responsabilité sociale des entreprises n'est pas assumée. Car, là où s'exercent les activités minières des recherches ou d'implantation, il règne « l'exploitation de l'homme par l'homme, le déséquilibre familial, l'immoralité, la pollution de l'environnement la délocalisation de nombreux villages et cimetières ${ }^{23}$.

Pour Amnesty International, les creuseurs, les artisanaux, ces mineurs qui reçoivent souvent très peu pour les minerais qu'ils extraient du sous-sol, sont victimes d'une exploitation généralisée en RDC, où les sites miniers sont contrôlés par des individus puissants, no-

21 Ces Elites sont ceux qui ont la mission de rappeler la phrase du CHRIST « Saul, Saul, pourquoi me persécute-tu?

22 FATSAH OUGUERGOUZ, Charte africaine de droit de l'Homme et des peuples. Une approche juridique des droits de l'Homme entre la tradition et la modernité, Ed.PUF, paris, 1993, p. IX.

23 MUHIGIRWA RUSEMBUKA F., Bonne gouvernance et secteur minier en R.D.Congo, Ed. CPAS,. 
tamment des responsables politiques et des groupes armés. Ils travaillent dans des conditions extrêmement dangereuses, généralement sans le moindre équipement de sécurité ${ }^{24}$.

Cette affirmation est d'une évidence incompréhensible dans les sites d'exploitation artisanale visités par nous. Mais pour notre part la responsabilité est mitigée. Est-ce une exploitation?

Il peut s'agir d'une auto-exploitation par le fait de l'ignorance. La recherche du gain facile a toujours des conséquences allant de la croyance à la psychopathologie dans le chef de celui qui ignore les principes de choix entre l'argent et la sagesse dans le travail.

La plupart de temps dans l'artisanat, comme le mot l'indique, c'est la méthode par tâtonnement qui prime dans la recherche des minerais. Les initiés sont aptes, mais ceux qui n'ont pas de connaissances soit scientifiques, soit empiriques du milieu ne peuvent que endurer dans leur apprentissage.

Souvent les apprentis vivent des déceptions qui leurs poussent, selon le niveau de l'éducation et de la culture, à des mauvais mécanismes de lutte contre les frustrations qui ne sont autres que la drogue, les indisciplines culturelles et spirituelles, les conclusions erronées encourageant au fétichisme.

Ce qui est drôle, c'est que tout le monde veut qu'on achète ses «bulongos », mais c'est de la terre de $2 \%$ de cuivre ou moins, au prix fixé par lui.

Dans ce cas de figure ce sont les négociants qui sont les plus perdants, ils sont tentés d'être malhonnêtes pensant gagner de cette manière alors que c'est doublement le contraire.

Et lorsqu'il est question d'une exploitation de l'homme par l'homme, les faiblesses matérielles, culturelles et institutionnelles soulèvent des inquiétudes sur la responsabilité de l'homme congolais ou étranger, de l'Etat-nation et de l'entreprise internationale du Congo.

De même les creusements ne gagnent pas non plus tant sur le plan économique que sanitaire et socio-psychologique.

Telle l'illustration en ces termes : «pour un sac de 185 Kilos, le chinois me donnent $72 \$$. Nous, les congolais, c'est pour nous tuer! $!^{25} »$

« Nous connaissons que ces chinois, avec leurs balances, ils nous volent beaucoup. Mais dans ce travail c'est comme cela que ça se $\operatorname{passe}^{26}$ » « si nous avions des dirigeants qui pouvaient nous aimer il serait impossible d'être pourchassés comme des étrangers dans notre propre pays! ${ }^{27}$ »

Il faut arriver à vendre ce pays et nous partager le prix pour que chacun puisse chercher où aller car il ne sert à rien ${ }^{28}$ 》).

24 AMNESTY INTERNATIONAL, Pertes et profits. Exploitation minière et droits humains dans le Katanga, en RDC, AMNESTY INTERNATIONAL, Londres, 2013.P.1.

25 MICHEL T. ET S., Katanga business, Ed. Double collector, Bruxelles, 2009.P.165.

26 Propos d'un négocient lors de notre échange avec lui sur la transparence dans la vente des minerais de cuivre à Kansonga..

27 Un creuseur de Kateketa nous confiait ce propos à Luisha.

28 Un groupe de Creuseurs et Négociants de Kambove criant sur nous après notre échange. 
Voilà le désespoir grandissant qui demeure dans leur être derrière tous ces attroupements de curiosité et de peur d'être chassés dans une carrière lorsqu'une autorité politique vient à leur rencontre qu'eux qualifient de baiser de Juda.

L'article 24 de la charte africaine de droits de l'homme et des peuples dispose que tous les peuples ont droit à un environnement satisfaisant et global, propice à leur développement.

On se pose des questions si l'Etat a perdu les repères de sa mission sacrée ou si les autorités politico-administratives se complaisent dans cette situation des choses. Est-ce impossible d'organiser et de responsabiliser cette ressource humaine?

Il faut analyser selon qu'il s'agit d'un creuseur, d'un négociant, d'une concession privée attribuée à un exploitant conformément à la loi, ou alors dans le cas d'une concession ouverte à l'exploitation minière artisanale. Ces lieux sont des poches des formations d'une sorte de criminalité sans pareil contre l'homme, l'Etat et l'environnement.

L'article 111 veut que seuls les Congolais majeurs puissent visiter et/exploiter les sites d'exploitation minière artisanale. Mais sur terrain la plupart de coopératives est le fruit des Chinois qui de temps en temps ont la possibilité de visiter leurs dépôts ouverts notoirement au nom des Congolais.

Il important de signaler que l'article $111 \mathrm{du}$ code minier n'aborde pas avec autant de clarté cette question comme nous l'abordons, mais c'est en combinant les termes d'éligibilité, de l'exposé des motifs et l'article 117 alinéa 2 y compris la pratique en la matière qu'un tel raisonnement peut avoir une justification. Ceci est par contre actuellement très tacite dans le projet de loi, venant du Gouvernement, portant code minier.

Sur le plan formel aucun problème de droit ne se pose. Là où le bas blesse c'est lorsqu'ils ont la possibilité de visiter les sites, ils ont non seulement la facilité de savoir comment rendre nul le bénéfice d'un pauvre Congolais mais aussi ils peuvent calculer le soidisant salaire de leurs journaliers à durée indéterminée en fonction des us et coutume de chez eux en y intégrant tous les extracontractuels. ${ }^{29}$

Le SAESSCAM est maintenant transformé, dans les carrières visitées, en un service de perception des frais d'autorisation de transport des minerais, la perception des cotisations des creuseurs et le règlement des différents par arrangement entre artisanaux avec autant des lacunes. L'encadrement en termes de techniques de travail n'est pas exécuté avec responsabilité ce qui est à la base de plusieurs accidents dans des carrières précitées.

Eux-mêmes, les agents de SAESSCAM ${ }^{30}$ sont à peine formés dans le domaine, l'unique ambition se place dans les entrailles de la survie sous forme d'un chômage déguisé. Pour avoir la carte de creuseur, la division des mines de la province du Katanga respecte la procédure dans le fait qu'il faut présenter l'attestation de bonne vie et mœurs. Le code de

29 Un Chinois disait à un Congolais avant d'aller dans le site qu'il pouvait, le Congolais,ne pas compter le salaire médiocre mais avoir beaucoup d'avantages parce qu'il peut aussi transporter et charger les camions de minerais à chaque sortie de la charge réalisée.

30 SAESSCAM, Service d'Assistance et d'encadrement du Small-Scale Mining. 
bonne conduite insiste aussi sur le respect de l'environnement. La question qui mérite d'être posée est celle de savoir pourquoi les sites des exploitants artisanaux, en dépit de la présence des postes de polices, des administrations publiques et les services spécialisés de renseignement, semblent être en dehors de l'Etat du Congo. L'Etat congolais n'est qu'un Etat occasionnel ${ }^{31}$ dans ses organes il convient de relever un sérieux problème de formation des animateurs des administrations publiques. Le SAESSCAM n'est alors qu'un service qui dénote d'un manque d'efficacité et d'efficience notoire dans les contextes de sites en questions.

De par l'analyse, l'exploitation minière artisanale fait partie de quatre formes d'exploitation minière organisées en RDC par le code minier.

Il s'agit de l'exploitation industrielle ${ }^{32}$, l'exploitation à petite ${ }^{33}$ échelle, l'exploitation de rejet $^{34}$ et l'exploitation minière artisanale ${ }^{35}$.Parmi les problèmes que soulève l'exploitation minière artisanale il y a aussi les techniques d'exploitation qui sont non conformes, l'absence de moyens de protection, l'absence d'encadrement technique des $\operatorname{artisanaux}^{36}$, le problème d'encadrement psycho-social, etc.

\section{LES PISTES DES SOLUTIONS}

Dans la plupart de nos interventions, nous abordons toujours la question sous l'angle institutionnel, psycho-social et de l'articulation des dispositions légales.

Dans la présente réflexion besoin est d'analyser notre position en soutenant le fait que le code minier, malgré ses imperfections dans sa conception, ne constitue pas le vrai problème. Bien que l'article 111 du code minier ne soit pas assorti d'une sanction pouvant protéger efficacement l'environnement mais fait appel à la conscience de ceux qui de temps en temps cherchent à neutraliser leurs consciences, ceci n'est pas fondamental.

La modification du code minier dans ses articles à problèmes est nécessaire mais pas déterminant. Parce que d'autres lois ${ }^{37}$ existent et peuvent combler les insuffisances du code minier lorsqu'il faut protéger l'environnement. C'est l'homme appelé à appliquer le code minier et d'autres lois connexes qui constitue le vrai problème. Cet homme vulnérable de-

31 Les organes appelés à représenter l'Etat congolais se comportent selon l'intérêt en présence. Lorsqu'ils sont devant une exigence de gouvernance, ils respectent les prescrits de l'administration (cas rare), mais souvent c'est en vertu des intérêts de survie (égoïstes) en justifiant cela par le fait de ne pas avoir un salaire digne.

32 Article 28 du code minier.

33 Article 97 du code minier.

34 Article 86 du code minier.

35 Article 26 du code minier.

36 ELENGI MOLAYI M., Législation minière, environnement et protection de la santé du travail des artisanaux miniers en R.D.Congo, Congo-Afrique XLVIII, Mai 2008 n425, P.373.

37 Il n'est pas interdit aux agent de l'environnement minier d'appliquer la loi portant principes fondamentaux relatifs à la protection de l'environnement, le code de la conservation de la nature de 2014 et les textes de nature internationale. 
puis sa naissance, sans repère spirituel et culturel, maltraité par l'étranger dans son histoire et sa vie présente, broyé par son Etat depuis son enfance, déséquilibré par le lux de la mondialisation, c'est celui-là qui doit appliquer les lois du pays sans une rémunération digne.

Est-ce les acteurs politiques savent que la gestion d'un peuple est une question de responsabilité tant nationale qu'internationale, de ce fait avec une possibilité de rendre compte? Cette question peut neutraliser toute sorte de réflexion tendant à proposer une espérance et une vision positive. Dès lors que le but de la recherche n'est pas de se faire écouter par les politiciens, qui ont déjà leurs calculs du pouvoir pour le pouvoir et non du pouvoir comme service, mais de faire évoluer la science, manquer de proposer des solutions serait impardonnable.

De ce qui précède, le code minier insiste sur le fait que seules les Congolais majeures peuvent avoir part et à l'entrée dans des sites d'exploitation minière artisanale et à l'activité de l'exploitation minière artisanale. Pour question de la dignité de la Nation congolaise à travers ses lois, il est important de faire respecter cette disposition. Le non respect de la dite disposition constitue l'une des causes poussant les Chinois à ne pas prendre au sérieux l'Etat congolais et les Congolais qui sont finalement utilisés comme journaliers à durée indéterminée.

Est-ce possible d'affirmer que les animateurs des institutions financières internationales se comportent en virus lorsqu'il s'agit d'assister techniquement les pays en développement?

C'est, à quelques nuances près, un sentiment de déception lorsqu'il est connu que les institutions de Breton Wood ont apporté une assistance technique lors de l'élaboration du code minier. Quelle mauvaise foi? Pour arriver à une exploitation minière artisanale respectueuse du développement socio-économique et environnemental de la RDC, le champ de notre investigation nous renseigne que outre la Direction de l'environnement minier dans ses ramifications et compétences, le SAESSCAM doit passer à une étape de l'adaptation rentable, non pas à travers des séminaires vulgaires et superficiels de renforcement des capacités mais par une amélioration qualitative.

Du fait que c'est l'institution légalement établie, il est nécessaire de recruter des personnes bien formées et ayant des valeurs de la vie humaine et non de la mort animale. Cette mentalité d'humain créé à l'image de Dieu doit d'abord se retrouver dans l'esprit de celui qui doit initier cette démarche pour que cela soit une réussite.

Dans nos démarches, besoin est d'arriver à une désintoxication spirito-scientifique pour un renouveau de l'homme congolais pour avoir un type d'animateur politico-administratif apte à comprendre les enjeux humanitaires, géostratégiques et géopolitiques de l'Etat du Congo. De ce fait, aucune solution efficace ne peut être envisageable dans cette confusion des pouvoirs démontrée par plusieurs crises constitutionnelles qu'a connues l'Etat du Congo.

Le phénomène creuseurs n'est qu'une expression de la vulnérabilité gouvernementale qui attire plusieurs autres types de vulnérables (hommes désespérés, femmes, enfants, prostitués, animateurs administratifs non encadrés,...). 
Du fait que l'Etat du Congo est actuellement conçu comme une maison construite à base d'un four à briques ratées, une sensibilisation scientifique doit être menée dans l'optique d'un conditionnement envisagé dans un lavage des cerveaux contre les pièges de l'époque léopoldienne, belge, mobutienne et la nouvelle gouvernance en tâtonnement.

Cette approche emporte l'exercice du pouvoir politique selon la mission sacrée de l'Etat du Congo, la bonne articulation des dispositions légales en la matière, le bon fonctionnement des administrations publiques, l'encadrement des creuseurs et groupes vulnérables, la vigilance des services des finances publiques, l'application de la loi portant principes fondamentaux relatifs à la protection de l'environnement, la création des banques efficaces et appropriées pour générer les emplois (encadrement des creuseurs...), la réhabilitation de la société congolaise dans ses valeurs, l'encouragement des modèles, etc.

En agissant de la sorte il est possible de rétablir l'Etat dans ses droits mais surtout dans ses obligations envers l'homme source première de son existence. Ainsi dans la pratique, les principes de traçabilité ${ }^{38}$ des minerais peuvent diminuer les opportunités des minerais de sang et d'exploitation de l'homme par l'homme.

Le contrôle des finances publiques peut être allégé, ce qui faciliterait la sécurité environnementale, économique et sociale de l'homme par la recherche du bonheur pour tous.

\section{CONCLUSION}

L'exploitation minière artisanale affecte non seulement l'environnement, mais aussi les finances publiques, la santé publique, la santé des creuseurs, la dignité humaine (droit à un travail digne, une rémunération équitable).

Il n'est pas rare de constater l'existence des lois, des institutions, des conférences, des discours traitant la question cruciale de la protection de la société par la protection des creuseurs.

Il n'est pas aussi rare de constater que ces discours, conférence, lois, institutions ne jouent presque pas la fonction appropriée pour arriver à mettre et remettre de l'ordre dans la société.

Cette façon de voir les choses continue à nous préoccuper sans que nous puissions, précocement, aborder telle ou telle autre thèse.

Cependant, traitant de la question de savoir s'il s'agit d'un déficit juridique ou d'une exploitation de l'homme par l'homme il est démontrable que toutes les deux possibilités sont une réalité de terrain. En d'autres termes, non seulement il est question ici de l'exploitation de l'homme par l'homme mais aussi le manque d'instruction, de culture du bonheur et de sens de responsabilité envers sa propre personne et sa nature d'homme créé à l'image de Dieu consolide une auto-exploitation.

38 Le principe de traçabilité veut que soient signalées, sur les différentes sortes de minerais, leurs sources de provenance afin de sanctionner au niveau mondial ceux qui alimentent la haute criminalité internationale pouvant rendre compétente la Cour Pénale Internationale. 
La manière simpliste de nous questionner est, selon nous, l'articulation des dispositions en la matière tout en indiquant la grande influence de la capacité de l'Etat du Congo à vivre selon sa mission sacrée depuis sa conception, sa création, son organisation et son fonctionnement. Cette position insiste sur la thèse fondée sur la culture en profondeur qui peut apporter un plus sur la géopolitique, la primauté de l'éducation, la thèse de l'institutionnalisation réelle et le leadership.

Pour un rappel sur les causes lointaines, il est vrai que le virus qui joue au même moment le rôle d'élément toxique a été introduit tour à tour par Léopold II, la Belgique, le souverain congolais et les acteurs ou animateurs internationaux.

Pour une approche en termes de solution durable, selon nous, cela doit passer par la désintoxication spirito-scientifique, assurer la quiétude contre la vulnérabilité gouvernementale tout en sachant que la RDC est semblable à une maison construite à base d'un four à briques ratées. Cette expression peut rendre incompréhensible l'idée mais notre souci est de rendre solide le schéma en se fondant sur les sciences exactes quant aux notions de la stabilité et de la résistance des matériaux.

Malheureusement cette maison avec toutes ses imperfections, est appelée à protéger le reste des briques ratées.

Cette analyse n'est qu'une provocation des questionnements sans prétention d'avoir épuisé la question autour de l'exploitation minière artisanale au Katanga.

\section{BIBLIOGRAPHIE SELECTIVE}

1. La loi $\mathrm{n}^{\circ} 11 / 009 \mathrm{du} 09$ Juillet 2011 portant principes fondamentaux relatifs à la protection de l'environnement et la loi n¹4/003 du 11Février 2014 relative à la conservation de la nature.

2. Le code minier ou la loi ${ }^{\circ} \mathrm{OO} 7 / 2002$ du 11 juillet 2002 portant code minier. Y compris le projet de loi portant code minier.

3. La constitution de la République Démocratique de Congo du 18/ février 2006, journal officiel de la RDC, cabinet du président de la République numéros spéciale $47^{\circledR}$ année, Kinshasa, février 2006.

4. Le code forestier, le code agricole et le code foncier.

5. RAPHAELE Rivier, Droit international public, Ed.PUF, Paris,2012.

6. MOLE GBAMANZANGO J.F, Réflexion sur quelques déclarations relatives aux droits de l'Homme dans l'histoire, in Revue Africaine de Droit canonique, vol. I, $\mathrm{n}^{\circ} 1$. Avril 2007.

7. FATSAH OUGUERGOUZ, Charte africaine de droit de l'Homme et des peuples. Une approche juridique des droits de l'Homme entre la tradition et la modernité, Ed.PUF, paris, 1993.

8. MICHEL T. ET S., Katanga business, Ed. Double collector, Bruxelles, 2009.

9. MUHIGIRWA RUSEMBUKA F., Bonne gouvernance et secteur minier en R.D.Congo, Ed. CPAS, 2008. 
10. ELENGI MOLAYI M., Législation minière, environnement et protection de la santé du travail des artisanaux miniers en R.D.Congo, Congo-Afrique XLVIII, Mai $2008 n^{\circ} 425$.

11. AIEPOJUG ONIBOKUM, La gestion des déchets Urbains. Des solutions pour l'Afrique, Ed. CRDI- Karthala, paris, 2001.

12. KISHIBA FITULA Gilbert.,- La libération : De la révolution vers l'Etat de droit, $\underline{\text { In }}$ actes des journées scientifiques, 1996-1997, $2^{\text {èm E }} \mathrm{d}$. Faculté des lettres de L'UNILU, PUL, 1997. - cours de droit international public II : les organisations internationales. DEA, UNILU, 2013.

13. BANZA MALALE M., Cours des questions approfondies de droit constitutionnel, DEA à la faculté de droit de L'UNILU, 2012-2013.

14. BANZA MALALE MAKUTA, Les_aspects juridiques dans les enjeux de crises congolaises des origines à nos jours (1885-2006), Ed. PUC, Kinshasa, 2011.

15. PNUE, Etude sur la portée de la potentialité environnementale de la RDC, Kinshasa, le 10 Octobre 2011.

16. AMNESTY INTERNATIONAL, Pertes et profits. Exploitation minière et droits humains dans le Katanga, en RDC, AMNESTY INTERNATIONAL, Londres, 2013. 